\title{
Molecular Structure of Muscle Filaments Determined by Electron Microscopy
}

\author{
*Correspondence to: \\ Craig $\mathrm{R}$, \\ Tel: +1-508-856-2474 \\ Fax: +1-508-856-1033 \\ E-mail: roger.craig@umassmed.edu
}

Received November 27, 2017

Revised December 16, 2017

Accepted December 16, 2017

\author{
Roger Craig \\ Division of Cell Biology and Imaging, Department of Radiology, \\ University of Massachusetts Medical School, Worcester, MA 01655, USA
}

\begin{abstract}
Electron microscopy and X-ray diffraction have together played a key role in our understanding of the molecular structure and mechanism of contraction of muscle. This review highlights the role of electron microscopy, from early insights into thick and thin filament structure by negative staining, to studies of single myosin molecule structure, and finally to recent high-resolution structures by cryo-electron microscopy. Muscle filaments are designed for movement. Their labile structures thus present challenges to obtaining near-atomic detail, which are also discussed.
\end{abstract}

Key Words: Muscle, Thick filament, Thin filament, Cryo-electron microscopy, Threedimensional reconstruction

\section{INTRODUCTION}

Muscles contract through interaction between two sets of overlapping protein filaments, the thick (myosin) and thin (actin) filaments (Steven et al., 2016). Contraction is switched on and off, or its strength modulated, by regulatory components on these filaments. The molecular basis of contraction has been elucidated over the past 60 years by a combination of electron microscopy and low angle X-ray diffraction (henceforth EM/XRD). In early models of muscle, actin and myosin were thought to coexist in a set of continuous filaments, which folded up to produce shortening. This was shown to be wrong by EM/XRD, which revealed that actin and myosin were in separate, discontinuous, parallel filaments that overlapped each other (Holmes, 2004). In combination with light microscopy, EM/XRD showed that contraction occurred through a sliding of the thick past the thin filaments, without change in their lengths. This review highlights the major advances in our understanding of muscle filament structure by EM since that time, and how the insights obtained have helped provide a mechanistic understanding of contraction. This has culminated in recent cryo-EM observations of thick and thin filaments at near- atomic resolution.

\section{THIN FILAMENTS}

Thin filaments are polymers of actin, tropomyosin and troponin. The helical arrangement of actin subunits in thin filaments was first suggested by XRD (Selby \& Bear, 1956), then directly demonstrated by negative staining EM (Hanson \& Lowy, 1963; Huxley, 1963) (Fig. 1A). Actin monomers are arranged in a double-helical array, like two strings of beads twisted around each other (Fig. 1B). Tropomyosin and troponin function as a $\mathrm{Ca}^{2+}$-sensitive regulatory complex that switches thin filament activity off at low $\mathrm{Ca}^{2+}$ and on at high $\mathrm{Ca}^{2+}$ levels (Gordon et al., 2000). How does this work? Negative stain EM/XRD combined with three-dimensional (3D) reconstruction showed that 400-A long coiled-coil tropomyosin molecules extend end-to-end along each strand of actin (Fig. 1C), each molecule interacting with, and regulating, seven actin monomers (Gordon et al., 2000). One $\mathrm{Ca}^{2+}$-sensitive troponin binds to each tropomyosin. X-ray studies showed changes in the "second actin layer line" when muscle was activated, consistent with an azimuthal movement of the tropomyosin strand on its associated actin helix

(a) This is an open-access article distributed under the terms of the Creative Commons Attribution Non-Commercial License (http://creativecommons.org/licenses/by-nc/4.0) which permits unrestricted noncommercial use, distribution, and reproduction in any medium, provided the original work is properly cited.

Copyrights @ 2017 by Korean Society of Microscopy 

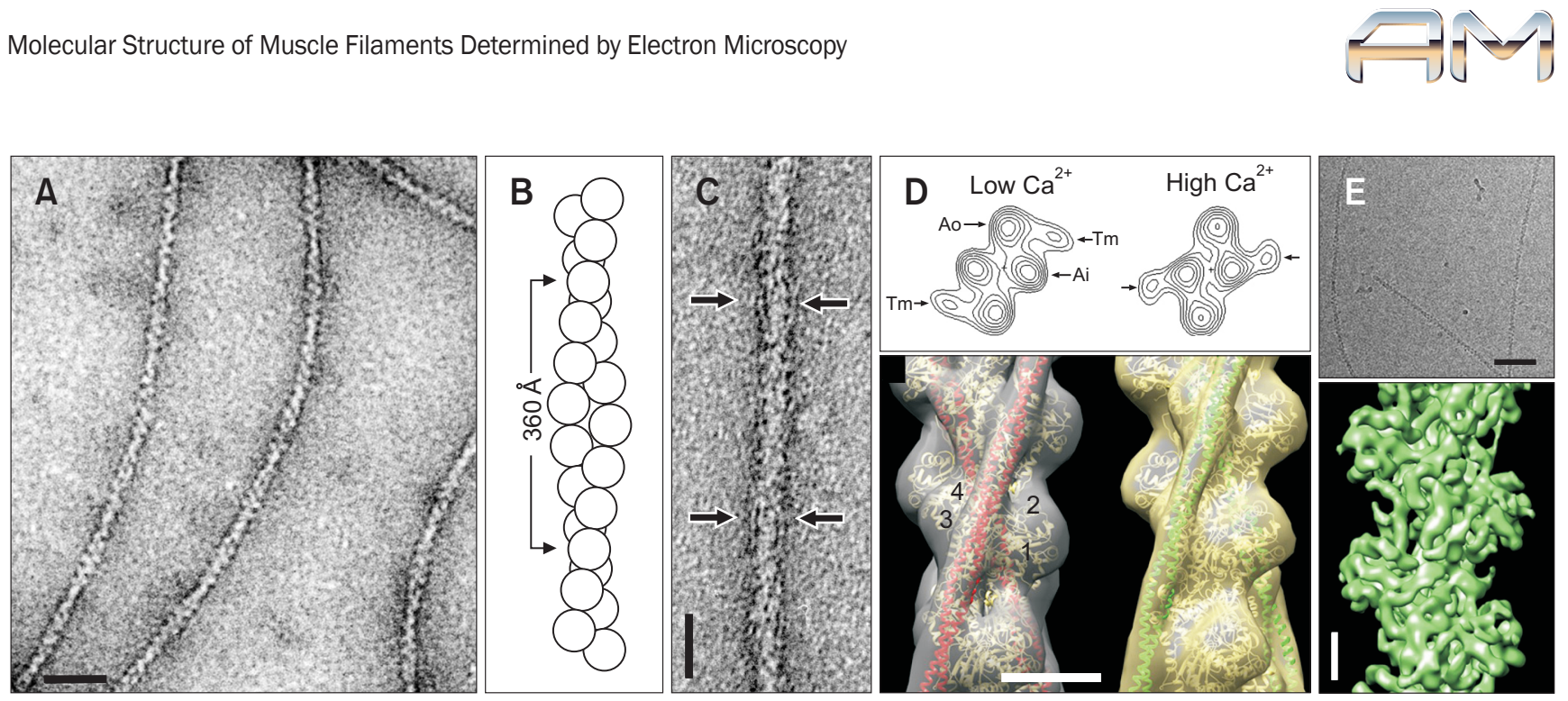

Fig. 1. Electron microscopy (EM) of thin filaments. (A) Negatively stained F-actin. (B) Model for F-actin based on EM. (C) Negatively stained thin filament showing tropomyosin strands (arrows) (from Moody et al., 1990, with permission). (D) Three-dimensional (3D) reconstruction of thin filaments based on negative stain data. Top, projection of 3D reconstruction down long pitch helices, showing two strands of actin subunits, each with an outer (Ao) and inner (Ai) domain (from Vibert et al., 1997, with permission). At low $\mathrm{Ca}^{2+}$, tropomyosin binds to the outer and in high $\mathrm{Ca}^{2+}$ to the inner domain. Bottom, surface views of reconstructions (grey) with fitting of actin atomic structure (yellow ribbon) and tropomyosin (red and green ribbons), showing leftward shift in tropomyosin from low $\mathrm{Ca}^{2+}$ (red) to high $\mathrm{Ca}^{2+}$ (green) (from Mun et al., 2014). (E) Cryo-EM of F-actin with direct detector, showing (top) excellent contrast and specimen preservation, even without stain, and (bottom) 3D reconstruction at $\sim 5.5 \AA \AA$ resolution, revealing actin secondary structure. Scale bars $=200 \AA$ (A, C); $50 \AA$ (D); $500 \AA$ (E, top), $20 \AA$ (E, bottom).

(Haselgrove, 1973; Huxley, 1973; Parry \& Squire, 1973). These studies suggested that at low $\mathrm{Ca}^{2+}$, tropomyosin lay on actin in a position that physically blocked myosin heads from binding to actin (the key requirement for generating the sliding force for contraction): thus, the steric blocking model of thin filament regulation was born. At high $\mathrm{Ca}^{2+}$, tropomyosin's azimuthal movement was thought to uncover the myosin binding site, allowing myosin head binding and contraction to ensue. This model was plausible and appealing, but, being based on modeling of X-ray patterns, was not definitive. EM images were needed. With improvements in negative staining, cryo-EM, and 3D reconstruction techniques, direct visual support for this model was eventually obtained (Lehman et al., 1994; Milligan et al., 1990). Tropomyosin strands were clearly seen on actin, and they were in different azimuthal positions at low and high $\mathrm{Ca}^{2+}$ (Fig. 1C and D; Mun et al., 2014). This was true of skeletal and cardiac filaments, and was independent of species (Lehman et al., 1995). With improvements in software for atomic fitting, it became possible to unambiguously dock the crystallographic structure of actin into the reconstructions (Fig. 1D). Based on this fitting, it was clear that tropomyosin in low $\mathrm{Ca}^{2+}$ did indeed cover key myosin binding sites on actin, and that its azimuthal movement across the actin strand at high $\mathrm{Ca}^{2+}$ uncovered most of these sites, sufficient to allow myosin binding (Vibert et al., 1997). Evidence from myosin-decorated filaments showed an additional movement of tropomyosin, caused by myosin binding itself, which would fully open the myosin binding site (Vibert et al., 1997). This suggested a 3-state mechanism of regulation, consistent with solution kinetics studies (McKillop \& Geeves, 1993).

Is this $20 \AA$ resolution structure, based on negative staining, correct? While uranyl acetate has been shown to be an excellent and rapid (<10 ms) fixative (Zhao \& Craig, 2003), its low $\mathrm{pH}(\sim 4 \mathrm{pH})$ and the specimen dehydration that occurs following staining could introduce artifacts. Cryo-EM, with its ability to capture near-native structure, free of staining and drying artifacts, and to reach near-atomic resolution (Fig. 1E), should help to answer this question. Recent cryoEM observations support different azimuthal positions of tropomyosin on actin in high resolution reconstructions (Harris et al., 2016; von der Ecken et al., 2015), suggesting that tropomyosin can indeed move; however, the low $\mathrm{Ca}^{2+}$ position seen by negative staining is not well supported in these studies. At low $\mathrm{Ca}^{2+}$, tropomyosin appears to occupy the position seen at high $\mathrm{Ca}^{2+}$ by negative stain, while at high $\mathrm{Ca}^{2+}$, tropomyosin is in the myosin-induced position (seen by negative stain). Cryo-EM is widely regarded as the gold standard for structural studies, and has recently produced numerous high resolution structures (Kuhlbrandt, 2014), including actin at $3.7 \AA$ resolution in these studies (von der Ecken et al., 2015). But could specimen preparation for cryo-EM introduce its own artifacts? With flexible or weakly interacting components (such as tropomyosin on actin), this can be an important issue. During cryo-grid preparation (sample application, blotting and plunging), filaments may bind to the carbon substrate (if one is used) or to the air-water interface, if filaments are viewed over 
holes. Either could potentially induce movement/disruption of tropomyosin. That such physical effects can occur is dramatically demonstrated in our lab's studies of myosin filaments. Myosin heads on native thick filaments are typically helically ordered when studied by negative staining, but are frequently disordered in cryo-EM images. The flexibility of head attachment to the filament makes for easy disruption of their ordered arrangement by physical forces (see THICK FILAMENTS). So which position of tropomyosin is correct (if either) — that observed by negative stain or cryo-EM? The answer is not yet clear. The blocked position observed by negative staining is supported by XRD of muscle (Poole et al., 2006), and by energy calculations (Orzechowski et al., 2014), which suggest that tropomyosin's interactions with actin are most stable when it is in the blocked position; binding is predicted to be weaker in the high $\mathrm{Ca}^{2+}$ position suggested by cryo-EM. Further study combined with ingenuity in experimental design is required to resolve this question.

\section{THICK FILAMENTS}

Thick filaments are bipolar polymers of myosin II molecules, with small amounts of accessory proteins (Craig \& Woodhead, 2006). Myosin II has two identical heavy chains that together form a long, coiled-coil tail in their C-terminal halves, while each folds into a globular head in its $\mathrm{N}$-terminal half; the two heads are flexibly attached to the tail and each is associated with two light chains (Fig. 2A). This molecular structure was first demonstrated by rotary shadowing EM (Fig. 2B) (Elliott \& Offer, 1978; Lowey et al., 1969). The arrangement of myosin molecules assembled into thick filaments was revealed by negative staining, soon after the invention of this technique (Huxley, 1963): the coiled-coil tails associated tightly to form the filament backbone, while the heads lay on its surface, where they could interact with actin to generate filament sliding and force (Fig. 2C). The heads in these early filament images were disordered, whereas XRD showed that in intact muscle they were actually ordered into helical or quasi-helical arrays (Huxley \& Brown, 1967; Wray et al., 1975). This discrepancy between EM and XRD was a further demonstration that some macromolecular complexes are quite labile and easily disturbed during preparation for EM (for example by binding to a hydrophilic grid surface; Knight \& Trinick, 1984), as discussed above for tropomyosin. Myosin
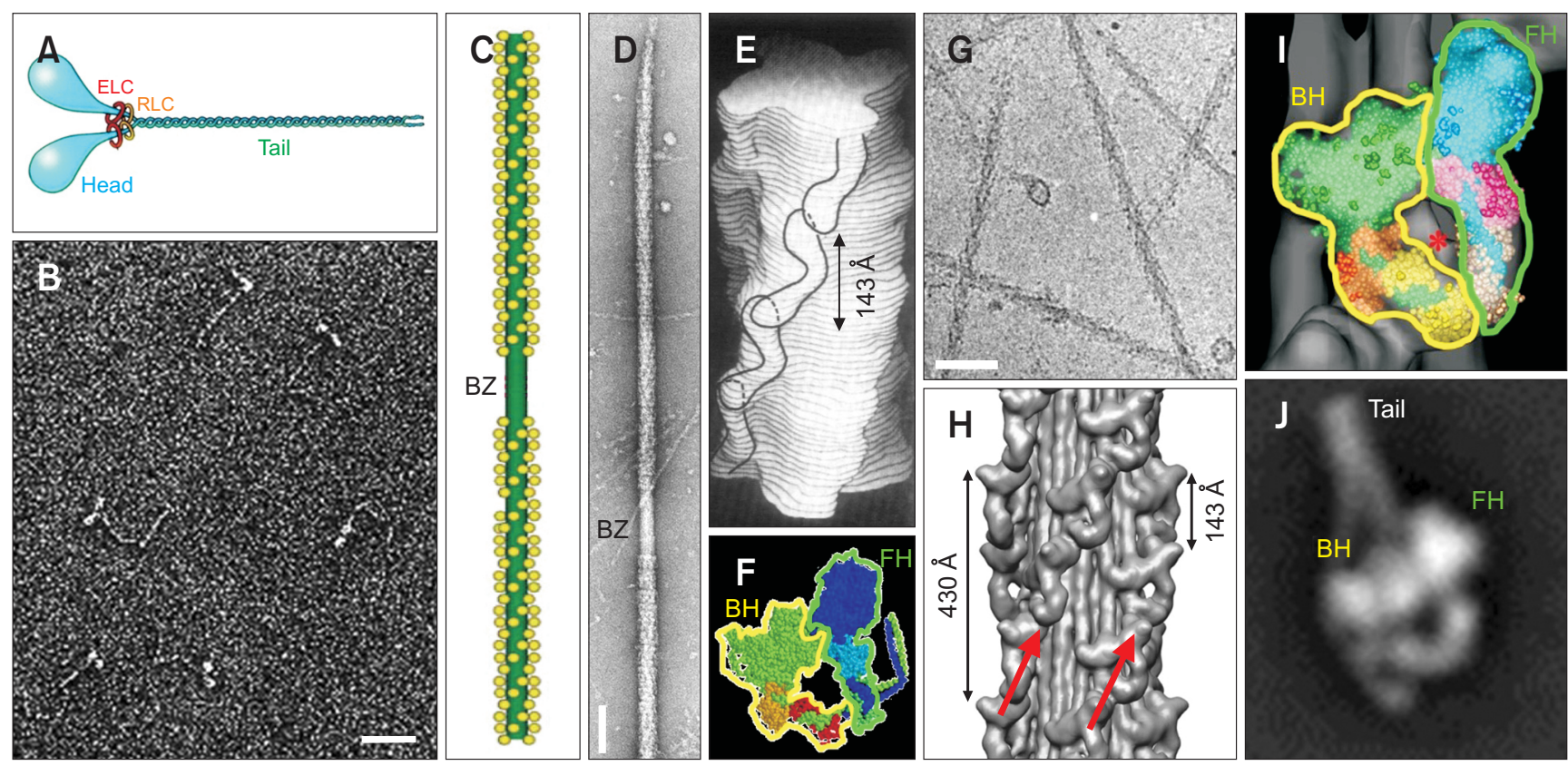

Fig. 2. Electron microscopy (EM) of myosin molecules and thick filaments. (A) Model for myosin II molecule. (B) Rotary shadowed myosin II at high salt, showing molecules with extended tail and two flexibly attached heads. (C) Model for thick filament, showing bipolar structure with helically ordered myosin heads. BZ, bare zone (a region of antiparallel overlap of myosin tails, lacking myosin heads). (D) Negative stain image of portion of thick filament from scallop striated muscle; helically ordered myosin heads produce regular pattern of staining. (E) Early thick filament model showing splayed heads, based on negatively stained tarantula thick filaments (based on Crowther et al., 1985, with permission). (F) Model for myosin interacting-heads structure based on cryo-EM tomography (based on Wendt et al., 2001), showing blocked and free heads (BH and FH). (G) Cryo-imaged tarantula thick filaments showing arrowhead appearance produced by helical ordering of heads (based on Woodhead et al., 2005). (H) Three-dimensional reconstruction of tarantula filament showing helical arrays (arrows) of J-shaped motifs representing pairs of myosin heads (based on Woodhead et al., 2005). (I) Fitting of myosin interacting-heads structure (F) to one J-structure of tarantula reconstruction, showing BH and FH (based on Woodhead et al., 2005). (J) Averaged image of smooth muscle myosin at physiological ionic strength with ATP present, showing folded tail and blocked and free heads. (B, D, G) Scale bars=1,000 Å. ELC, essential light chain; RLC, regulatory light chain. 
heads are designed to be mobile, as their primary function is to extend from the thick filament to actin and undergo a large conformational change (the power-stroke) that propels thin past thick filaments to produce contraction. This mobility is reflected in their flexible attachment to the tail. Unfortunately, this essential feature of myosin function leads to difficulties for EM preservation and thick filament 3D reconstruction, which requires ordered arrays.

The helical organization of heads known to be present from XRD was first directly observed by negative staining EM of invertebrate filaments, which have more stable helices than vertebrates (Stewart et al., 1981) (Fig. 2D). This breakthrough allowed the first 3D reconstruction of a thick filament (Stewart et al., 1981), with reconstructions of other species (including vertebrates) following, revealing a diversity of filament symmetries (Crowther et al., 1985; Stewart \& Kensler, 1986; Vibert \& Craig, 1983). While invertebrate filaments provided a critical breakthrough, the reconstructions had limited resolution and were difficult to interpret. The low resolution resulted from head mobility, present even in invertebrates, and from technical limitations of the Fourier-based helical reconstruction methods available at the time (Crowther et al., 1985). Most reconstructions were interpreted in terms of a splayed conformation of myosin heads, one pointing towards the tip of the filament and the other towards its center, such that heads from adjacent axial levels (i.e., from different myosin molecules) interacted with each other (Fig. $2 \mathrm{E})$. This interpretation was later shown to be fundamentally wrong, following advances in thick filament imaging and reconstruction, and in understanding the structure of single myosin molecules.

\section{MYOSIN MOLECULES}

The structure of myosin described earlier (long tail, two flexibly attached heads) was based on studies of the skeletal muscle protein at high ionic strength $(0.5 \mathrm{M}$ - used to dissolve thick filaments into their component molecules). A completely different conformation was observed when smooth muscle myosin molecules were examined, and conditions closer to physiological were used ( $0.15 \mathrm{M}$ salt, presence of Mg.ATP). Cryo-electron tomography of two-dimensional (2D) crystals of smooth muscle heavy meromyosin (a soluble, proteolytic fragment of myosin), showed that in the inhibited state, where myosin's ATPase activity is switched off (the equivalent of the relaxed state of muscle), the two myosin heads interacted with each other in an asymmetric arrangement (Wendt et al., 2001) (Fig. 2F). One head (the "blocked" head) had its actinbinding site blocked by binding to the other (the "free" head). The actin-binding region of the free head was not blocked, but binding of the blocked head to the free head converter domain (involved in mechano-chemical coupling), suggested that ATPase activity would be inhibited in the free head. This interacting-heads conformation provided a compelling explanation for the structural basis of the switched-off state of smooth muscle myosin, and why both heads are needed for inhibition of activity (Wendt et al., 2001).

\section{CRYO-EM OF THICK FILAMENTS}

Advances in EM imaging and 3D reconstruction, combined with these new insights into myosin molecule conformation, led to a critical breakthrough in our understanding of thick filament structure (Woodhead et al., 2005). Cryo-EM of tarantula thick filaments (used because they have an especially stable array of myosin heads) showed excellent preservation of helical order; Fig. 2G). A new and powerful method of helical analysis, Iterative Helical Real Space Reconstruction, produced a $25 \AA$ resolution 3D reconstruction (Fig. 2H), showing unambiguously resolved pairs of myosin heads for the first time. When the interacting-heads atomic model of smooth muscle myosin molecules (Fig. 2F) was docked into the reconstruction, a perfect fit was obtained (Woodhead et al., 2005) (Fig. 2I). This demonstrated beyond any doubt that heads were not splayed (Crowther et al., 1985), but in fact interacted with each other in thick filaments as they do in single molecules. This configuration immediately suggested how thick filaments are switched off in relaxed musclejust as in single molecules-by head-head interactions. In filaments, additional interactions were also seen. One was between the blocked head and the first part of the myosin tail, onto which this head was folded; others involved interactions between different myosin molecules. These multiple, weak interactions would serve to prevent heads in thick filaments both from hydrolyzing ATP and interacting with actin. Subsequent studies showed similar interactions in a variety of other invertebrates as well as in vertebrate muscle (Woodhead et al., 2013; Zoghbi et al., 2008). It is apparent that headhead interaction is an essentially universal mechanism for inhibiting myosin filament activity in relaxed muscle across the animal kingdom. The interacting heads structure is also supported by X-ray and fluorescence polarization studies of intact striated muscle (Fusi et al., 2015; Reconditi et al., 2011), confirming that it is not an artifact of filament isolation or EM preparative procedures.

\section{MYOSIN MOLECULES UNDER PHYSIOLOGICAL CONDITIONS}

A missing feature of the interacting-heads structure seen in the 2D crystals of myosin molecules was the myosin tail, which was not clearly visualized in reconstructions of either heavy meromyosin or full length myosin (Wendt et al., 2001). Early studies by rotary shadowing EM showed that myosin in 
the off-state had a compact structure, in which the tail folded into three segments running parallel to each other (Onishi \& Wakabayashi, 1982); the two heads bent back towards the folded-tail, but head-head interaction was not observed, probably due to disruption of the interactions by the high negative charge of the mica on which molecules are deposited for rotary shadowing. The structure of myosin showing both interacting heads and the folded tail was revealed following advances in single molecule negative staining (Burgess et al., 2004). Routine negative staining with uranyl acetate works well for large structures (filaments, viruses, etc.) but not with small, individual protein molecules. A key advance was to treat the grid surface with UV radiation or plasma discharge, making the carbon surface highly hydrophilic. This enables the stain to spread in a very thin layer, such that individual heads and even the $20 \AA$-diameter tail could be seen. Single molecules imaged in this way showed the tri-folded tail and in addition the interacting heads (as in the $2 \mathrm{D}$ crystals and filaments) (Burgess et al., 2007). 2D averaging of these molecules shows that the myosin tail wraps around the blocked head in this compact structure, which would likely add to the inhibitory effect of the head-head interactions (Fig. 2J) (Burgess et al., 2007).

Single molecule EM studies have shown that this folded, interacting-heads structure is present in myosin II molecules from smooth, skeletal, cardiac and nonmuscle cells, across the animal kingdom, from vertebrates to sea anemones (the most primitive animals with muscles) (Jung et al., 2008; Lee et al., 2016). Like the interacting-heads structure in filaments, it appears to be an essentially universal conformation for myosin in its single molecule form under approximately physiological conditions. This compact, inhibited structure appears to be nature's mechanism for storing or transporting myosin II in all cells, including smooth, striated and nonmuscle cells (Cross, 1988). EM shows that this inhibited structure is switched on by $\mathrm{Ca}^{2+}$-induced phosphorylation of the regulatory light chain in the myosin head (Craig et al., 1983). This causes breakage of the head-head interactions and unfolding of the tail, allowing molecules to assemble into filaments that can interact with actin and generate cell motility.

\section{FUTURE STUDIES}

The current resolution revolution in cryo-EM (Kuhlbrandt, 2014) opens the possibility of obtaining near-atomic detail in both thick and thin filaments and myosin molecules. However, unlike many molecules that have been solved to 3 $\AA$ resolution or better, these filaments and molecules present difficulties due to their labile nature. While actin in thin filaments has been resolved to $3.7 \AA$, tropomyosin resolution is lower $(6.5 \AA)$, presumably due to its mobility and the non-identical interactions it makes with actin at each of its seven sites of contact (von der Ecken et al., 2015). Whether tropomyosin can be stabilized to yield more detail is not clear. In addition, as discussed earlier, even high resolution will have limited value for understanding thin filament regulation if tropomyosin's position is in doubt due to possible preparative artifacts. Similar problems exist with myosin. The highest resolution obtained for tarantula thick filaments so far is $13 \AA$ (Yang et al., 2016). This should improve with a direct detector, although head mobility in the specimen itself may limit resolution. We do not yet know how great this limitation will be. Thick filaments from insect flight muscle have been resolved to $6 \AA$ (for the myosin tails in the backbone), showing that high resolution can be obtained, but resolution of the heads is significantly lower, again presumably due to their mobility (Hu et al., 2016). Single myosin molecules are also labile and their structure easily disrupted. It remains to be seen how much this will limit resolution and whether a nearatomic structure can be obtained.

\section{CONFLICT OF INTEREST}

No potential conflict of interest relevant to this article was reported.

\section{ACKNOWLEDGMENTS}

This work was supported by grants from the NIH (AR062279, AR067279, and AR072036). I thank members of my lab (Drs. John Woodhead, Kyoung Hwan Lee and Shixin Yang) for numerous discussions of the issues presented here, Dr. Lee for the cryo image in Fig. 1E, and Dr. Yang for the reconstruction in Fig. 1E and image average in Fig. 2J.

\section{REFERENCES}

Burgess S A, Walker M L, Thirumurugan K, Trinick J, and Knight P J (2004) Use of negative stain and single-particle image processing to explore dynamic properties of flexible macromolecules. J. Struct. Biol. 147, 247-258.
Burgess S A, Yu S, Walker M L, Hawkins R J, Chalovich J M, and Knight P J (2007) Structures of smooth muscle myosin and heavy meromyosin in the folded, shutdown state. J. Mol. Biol. 372, 1165-1178.

Craig R, Smith R, and Kendrick-Jones J (1983) Light-chain phosphory- 
Iation controls the conformation of vertebrate non-muscle and smooth muscle myosin molecules. Nature 302, 436-439.

Craig R and Woodhead J L (2006) Structure and function of myosin filaments. Curr. Opin. Struct. Biol. 16, 204-212.

Cross R A (1988) What is 10 S myosin for? J. Muscle Res. Cell Motil. 9 108-110.

Crowther R A, Padron R, and Craig R (1985) Arrangement of the heads of myosin in relaxed thick filaments from tarantula muscle. J. Mol. Biol. 184, 429-439.

Elliott A and Offer G (1978) Shape and flexibility of the myosin molecule. J. Mol. Biol. 123, 505-519.

Fusi L, Huang Z, and Irving M (2015) The conformation of myosin heads in relaxed skeletal muscle: implications for myosin-based regulation. Biophys. J. 109, 783-792.

Gordon A M, Homsher E, and Regnier M (2000) Regulation of contraction in striated muscle. Physiol. Rev. 80, 853-924.

Hanson J and Lowy J (1963) The structure of F-actin and of actin isolated from muscle. J. Mol. Biol. 6, 46-60.

Harris S P, Belknap B, Van Sciver R E, White H D, and Galkin V E (2016) $\mathrm{CO}$ and $\mathrm{C} 1 \mathrm{~N}$-terminal Ig domains of myosin binding protein $\mathrm{C}$ exert different effects on thin filament activation. Proc. Natl. Acad. Sci. U S A 113, 1558-1563.

Haselgrove J C (1973) X-ray evidence for a conformational change in the actin-containing filaments of vertebrate striated muscle. Cold Spr. Harbor Symp. Quant. Biol. 37, 341-352.

Holmes K C (2004) Introduction. Philos. Trans. R. Soc. Lond. B Biol. Sci. 359, 1813-1818.

Hu Z, Taylor D W, Reedy M K, Edwards R J, and Taylor K A (2016) Structure of myosin filaments from relaxed Lethocerus flight muscle by cryo-EM at 6 A resolution. Sci. Adv. 2, e1600058.

Huxley H E (1963) Electron microscope studies on the structure of natural and synthetic protein filaments from striated muscle. J. Mol. Biol. 7, 281-308.

Huxley H E (1973) Structural changes in the actin- and myosin-containing filaments during contraction. Cold Spr. Harbor Symp. Quant. Biol. 37, 361-376.

Huxley H E and Brown W (1967) The low-angle x-ray diagram of vertebrate striated muscle and its behaviour during contraction and rigor. J. Mol. Biol. 30, 383-434.

Jung H S, Komatsu S, Ikebe M, and Craig R (2008) Head-head and headtail interaction: a general mechanism for switching off myosin II activity in cells. Mol. Biol. Cell 19, 3234-3242.

Knight $\mathrm{P}$ and Trinick J (1984) Structure of the myosin projections on native thick filaments from vertebrate skeletal muscle. J. Mol. Biol. 177, 461-482.

Kuhlbrandt W (2014) Biochemistry. The resolution revolution. Science 343, 1443-1444.

Lee K H, Yang S, Liu X, Korn E D, Sarsoza F, Bernstein S I, Pollard L, Lord M J, Trybus K M, and Craig R (2016) Myosin II head interaction in primitive species. Biophys. J. 110 (Suppl 1), 615a.

Lehman W, Craig R, and Vibert P (1994) Ca2+-induced tropomyosin movement in Limulus thin filaments revealed by three-dimensional reconstruction. Nature 368, 65-67.

Lehman W, Vibert P, Uman P, and Craig R (1995) Steric-blocking by tropomyosin visualized in relaxed vertebrate muscle thin filaments. J. Mol. Biol. 251, 191-196.
Lowey S, Slayter H S, Weeds A G, and Baker H (1969) Substructure of the myosin molecule. I. Subfragments of myosin by enzymic degradation. J. Mol. Biol. 42, 1-29.

McKillop D F and Geeves M A (1993) Regulation of the interaction between actin and myosin subfragment 1: evidence for three states of the thin filament. Biophys. J. 65, 693-701.

Milligan R A, Whittaker M, and Safer D (1990) Molecular structure of F-actin and location of surface binding sites. Nature 348, 217-221.

Moody C, Lehman W, and Craig R (1990) Caldesmon and the structure of smooth muscle thin filaments: electron microscopy of isolated thin filaments. J. Muscle Res. Cell Motil. 11, 176-185.

Mun J Y, Previs M J, Yu H Y, Gulick J, Tobacman L S, Beck P S, Robbins J, Warshaw D M, and Craig R (2014) Myosin-binding protein C displaces tropomyosin to activate cardiac thin filaments and governs their speed by an independent mechanism. Proc. Natl. Acad. Sci. U S A 111, 2170-2175.

Onishi H and Wakabayashi T (1982) Electron microscopic studies of myosin molecules from chicken gizzard muscle I: the formation of the intramolecular loop in the myosin tail. J. Biochem. 92, 871-879.

Orzechowski M, Moore J R, Fischer S, and Lehman W (2014) Tropomyosin movement on F-actin during muscle activation explained by energy landscapes. Arch. Biochem. Biophys. 545, 63-68.

Parry D A and Squire J M (1973) Structural role of tropomyosin in muscle regulation: analysis of the X-ray diffraction patterns from relaxed and contracting muscles. J. Mol. Biol. 75, 33-55.

Poole K J, Lorenz M, Evans G, Rosenbaum G, Pirani A, Craig R, Tobacman L S, Lehman W, and Holmes K C (2006) A comparison of muscle thin filament models obtained from electron microscopy reconstructions and low-angle X-ray fibre diagrams from non-overlap muscle. J. Struct. Biol. 155, 273-284.

Reconditi M, Brunello E, Linari M, Bianco P, Narayanan T, Panine P, Piazzesi G, Lombardi V, and Irving M (2011) Motion of myosin head domains during activation and force development in skeletal muscle. Proc. Natl. Acad. Sci. U S A 108, 7236-7240.

Selby C C and Bear R S (1956) The structure of actin-rich filaments of muscles according to x-ray diffraction. J. Biophys. Biochem. Cytol. 2, 71-85.

Steven A C, Baumeister W, Johnson L N, and Perham R N (2016) Molecular Biology of Assemblies and Machines (Garland Science, New York and London).

Stewart M and Kensler R W (1986) Arrangement of myosin heads in relaxed thick filaments from frog skeletal muscle. J. Mol. Biol. 192, 831-851.

Stewart M, Kensler R W, and Levine R J (1981) Structure of Limulus telson muscle thick filaments. J. Mol. Biol. 153, 781-790.

Vibert P and Craig R (1983) Electron microscopy and image analysis of myosin filaments from scallop striated muscle. J. Mol. Biol. 165, 303320.

Vibert P, Craig R, and Lehman W (1997) Steric-model for activation of muscle thin filaments. J. Mol. Biol. 266, 8-14.

Von Der Ecken J, Muller M, Lehman W, Manstein D J, Penczek P A, and Raunser S (2015) Structure of the F-actin-tropomyosin complex. Nature 519, 114-117.

Wendt T, Taylor D, Trybus K M, and Taylor K (2001) Three-dimensional image reconstruction of dephosphorylated smooth muscle heavy meromyosin reveals asymmetry in the interaction between myosin heads and placement of subfragment 2. Proc. Natl. Acad. Sci. U S A 


\section{8, 4361-4366.}

Woodhead J L, Zhao F Q, and Craig R (2013) Structural basis of the relaxed state of a $\mathrm{Ca} 2+$-regulated myosin filament and its evolutionary implications. Proc. Natl. Acad. Sci. U S A 110, 85618566.

Woodhead J L, Zhao F Q, Craig R, Egelman E H, Alamo L, and Padron $R$ (2005) Atomic model of a myosin filament in the relaxed state. Nature 436, 1195-1199.

Wray J S, Vibert P J, and Cohen C (1975) Diversity of cross-bridge confi- gurations in invertebrate muscles. Nature 257, 561-564.

Yang S, Woodhead J L, Zhao F Q, Sulbaran G, and Craig R (2016) An approach to improve the resolution of helical filaments with a large axial rise and flexible subunits. J. Struct. Biol. 193, 45-54.

Zhao F Q and Craig R (2003) Capturing time-resolved changes in molecular structure by negative staining. J. Struct. Biol. 141, 43-52.

Zoghbi M E, Woodhead J L, Moss R L, and Craig R (2008) Threedimensional structure of vertebrate cardiac muscle myosin filaments. Proc. Natl. Acad. Sci. U S A 105, 2386-2390. 